\title{
Thyroid function and IVF outcome for different indications of subfertility
}

C C Repelaer van Driel-Delprat1, E W C M van Dam², P M van de Ven³, K Aissa1, M K ter Haar1, Y Feenstra1, A de Roos' ${ }^{1}$, G Beelen ${ }^{1}$, R Schats ${ }^{1}$ and C B Lambalk ${ }^{1}$

1Division of Reproductive Medicine, Department of Obstetrics, Gynaecology and Reproductive Medicine, Amsterdam Reproduction \& Development, Amsterdam UMC, Vrije Universiteit Amsterdam, Amsterdam, Netherlands

2Division of Endocrinology, Department of Internal Medicine, Amsterdam UMC, Vrije Universiteit Amsterdam, Amsterdam, Netherlands

${ }^{3}$ Department of Epidemiology and Biostatistics, Amsterdam UMC, Vrije Universiteit Amsterdam, Amsterdam, Netherlands

Correspondence should be addressed to C C Repelaer van Driel-Delprat: C.Delprat@amsterdamumc.nl

Graphical Abstract

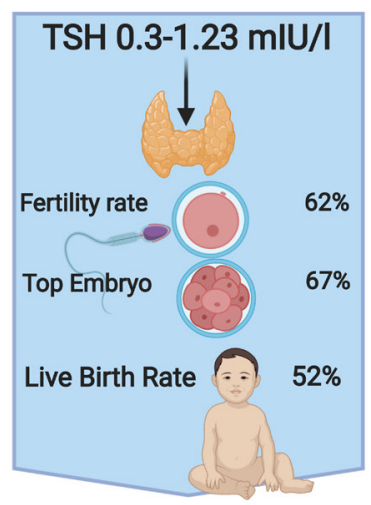

\section{Subfertile IVF-treated Women $n=949$}

\begin{tabular}{l|c|c|r|r|} 
& \multicolumn{3}{|c}{ TSH (mlU/L) } \\
primary & $49.8 \%$ & $0.3-1.21$ & $50.2 \%$ & secondary \\
subfertile & $47.5 \%$ & $1.22-1.68$ & $52.5 \%$ & subfertile \\
women & $48.1 \%$ & $1.69-2.31$ & $51.9 \%$ & women \\
\hline & $60.9 \%$ & $2.32-4.5$ & $39.9 \%$ & \\
& & &
\end{tabular}

Primary Subfertile IVF-treated Women $n=489$
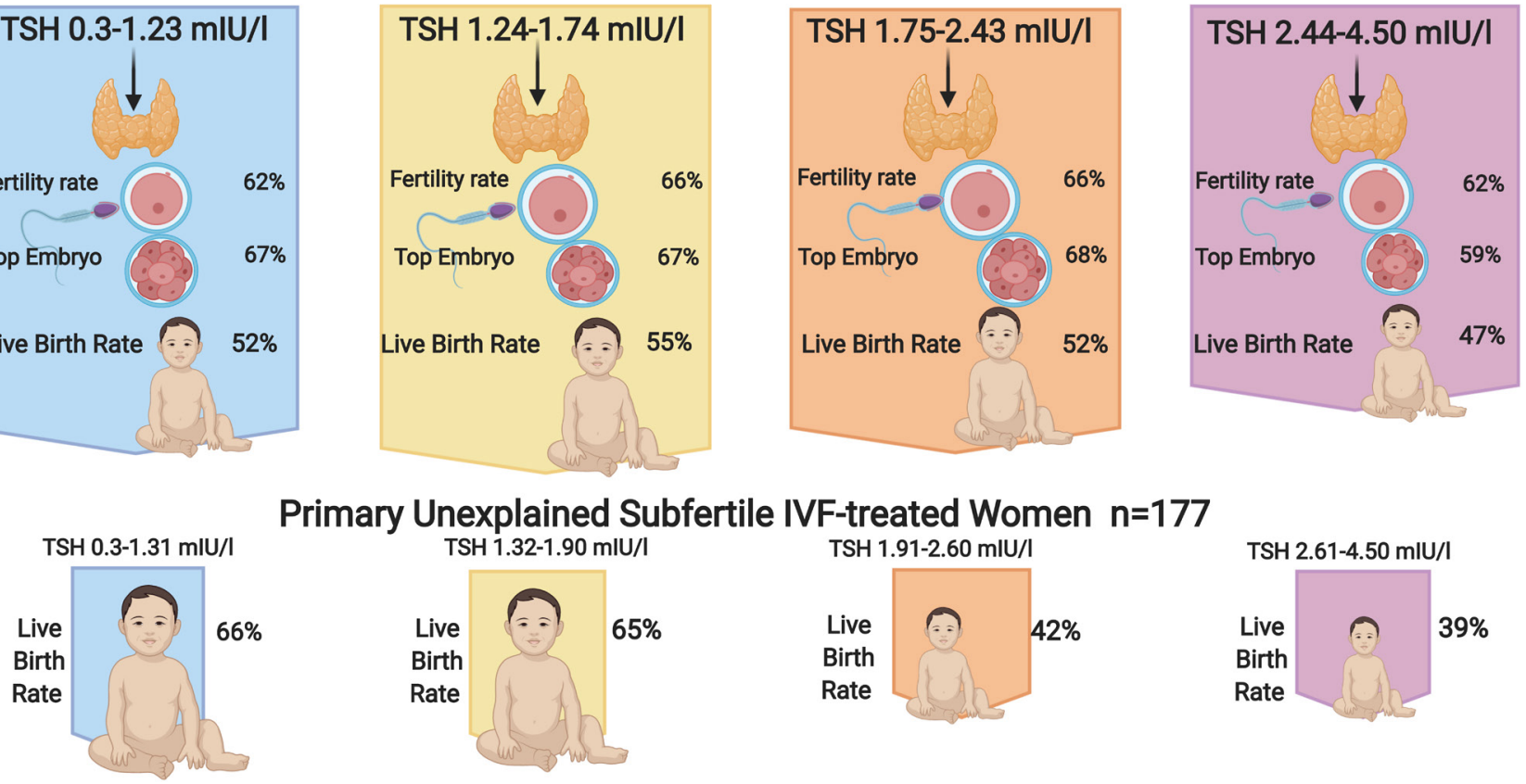

Primary Unexplained Subfertile IVF-treated Women $n=177$
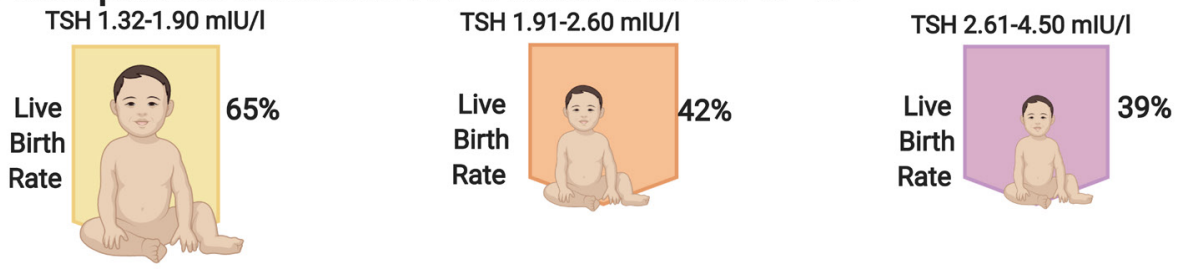


\begin{abstract}
Studies evaluating pregnancy outcomes after assisted reproductive treatment (ART) in women with high-normal (2.5-4.5 mIU/L) thyroid-stimulating hormone (TSH) levels are conflicting, possibly due to different patient charactistics and subfertility indications. The aim of this study was to examine the hypothesis that high-normal compared to low-normal TSH levels are associated with adverse implications for pregnancy outcomes in conventional in vitro fertilization (IVF)treated women. Therefore, we analyzed retrospectively the characteristics and pregnancy outcomes of 949 subfertile women with TSH 0.3-4.5 mIU/L, treated with conventional IVF between January 2008 and March 2012. Demographic and baseline characteristics were compared between groups of patients based on TSH quartiles, using one-way Anova, Kruskal-Wallis ANOVA and chi-square test. Women with high-normal quartile TSH were significantly more likely to be primary subfertile $(P=0.01)$, with a higher prevalence of unexplained subfertility and with $15 \%$ fewer live births after IVF compared to lower TSH quartiles $(P=0.02)$. In secondary subfertile women with high-normal TSH, male factor subfertility prevailed $(P=0.01)$, with more live births $(P=0.01)$. When analyzing primary and secondary subfertile women as one group, these differences failed to be observed, showing no differences in cumulative pregnancy outcomes of IVF between TSH quartiles (I: 0.3-1.21 mIU/L; II: 1.22-1.68 mIU/L; III: 1.69-2.31 mIU/L; IV: 2.32-4.5 mIU/L). In conclusion, primary subfertile women predominate in the high-normal TSH quartile, associated with significantly fewer live births in a subgroup of primary unexplained subfertile women $(9 \% ; n=87 / 949)$, while in secondary subfertile women, dominated by male factor subfertility, high-normal TSH is associated with more live births.
\end{abstract}

\title{
Lay summary
}

Thyroid hormones are required for all cell processes in the body. An underactive thyroid gland, in which insufficient thyroid hormones are produced and thyroid-stimulating hormone (TSH) rises, is associated with a lower chance of pregnancy. It is not yet clear above which TSH level, 4.5 or also $2.5 \mathrm{mIU} / \mathrm{L}$, this lower probability occurs. Therefore, in 949 couples treated with conventional IVF, we examined whether high-normal TSH levels (TSH: 2.5-4.5 mIU/L) compared to low normal TSH levels (0.3-2.5 mIU/L) affect the live birth rate. We found that women who were trying to become pregnant for the first time, especially without any other cause, that is unexplained subfertility, were more likely to have higher TSH levels. These women had a much lower chance of having a baby compared to women with low-normal TSH levels.

Keywords: - thyroid-stimulating hormone

- conventional in vitro fertilization

- primary subfertility

- unexplained subfertility

- live birth rate

Reproduction and Fertility (2021) 2 280-291

\section{Introduction}

Subclinical hypothyroidism, defined as TSH above the reference range with FT4 within the reference range, affects $2-4 \%$ of women of fertile age (Baloch et al. 2003). The upper reference level of TSH for subfertile women is a matter of debate, set at $4.5 \mathrm{mIU} / \mathrm{L}$ according to the American Society for Reproductive Medicine (Practice Committee of the American Society for Reproductive 2015) but at $2.5 \mathrm{mIU} / \mathrm{L}$ according to the American Thyroid Association (Alexander et al. 2017). Thyroid hormone levels affect oocyte quality and ovulation (Zhang et al. 2013) by interaction with FSH on the granulosa cells and on LH/ hCG formation in rats. In humans, lower donor TSH levels are positively associated with recipient clinical pregnancy, indicating influence at the level of the oocyte (Karmon et al. 2016). Fertilization and embryo quality are lower in women with higher TSH (Cramer et al. 2003), though others could not demonstrate this, as reflected in pregnancy rates (Reh et al. 2010, Chai et al. 2014, Alexander et al. 2017).

Reports on intrauterine insemination (IUI)populations (Jatzko et al. 2014, Karmon et al. 2015, Unuane et al. 2017, Tuncay et al. 2018, Pekcan et al. 2019) including our own (Repelaer van Driel-Delprat et al. 2019) do not support lowering the TSH upper limit of normal for IUItreated subfertile women.

Reports on the optimal TSH upper normal limit in women undergoing conventional in vitro fertilization (IVF) or intracytoplasmatic sperm injection (ICSI) vary concerning associated live birth rate, clinical pregnancy, pregnancy loss, fertilization rate and embryo quality (Zhong 2012, Zhao et al. 2018). Overall, because of different

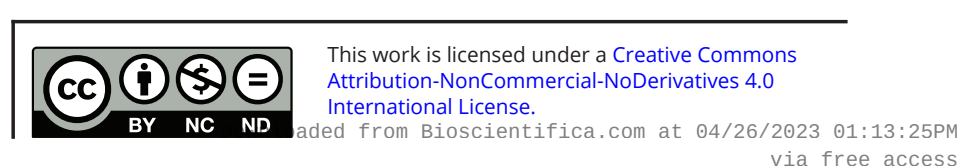


study designs, general conclusions are difficult to draw, due to various TSH upper references levels used (from 2.5 to $5 \mathrm{mIU} / \mathrm{L}$ ) as well as the different primary endpoints such as live birth rate or loss of pregnancy or embryo quality. In addition, the investigated cohorts were always a mix of women treated with conventional IVF or with ICSI, each with their typical subfertility indications, or involved ICSI-treated women only (Poppe et al. 2018b). In the Netherlands, ICSI is applied predominantly for severe male factor and after total fertilization failure with conventional IVF, while in many countries, ICSI is routinely used for all indications. As the fertilization process is basically different in conventional IVF and ICSI, and with the possible known impact of thyroid hormones on fertilization or early embryo development, we aimed to analyze only conventional IVF and its various subfertility indications. Primary and secondary subfertile women were so far not analyzed separately, while secondary subfertility is associated with an increased prevalence of thyroid autoimmunity, disappearing after correction for age (Tan et al. 2014), and in fecund women, no association of highnormal TSH levels and subfertility levels is observed.

In the tablet trial, $20.1 \%$ of subfertile euthyroid women have TSH levels $>2.5 \mathrm{mIU} / \mathrm{L}$ (Dhillon-Smith et al. 2020) as do $20-26 \%$ of women treated with ART (Reh et al. 2010, Michalakis et al. 2011). Classifying TSH values above $2.5 \mathrm{mIU} / \mathrm{L}$ as subclinical hypothyroidism might lead to a disproportionally increased prevalence in subfertile women, likely contributing to overdiagnosis (Reh et al. 2010) and a burden to the health care systems (DhillonSmith et al. 2020).

Therefore, the aim of this study was to examine the hypothesis that in conventional IVF-treated women, excluding ICSI, high-normal compared to low-normal TSH levels are associated with different pregnancy outcomes.

\section{Patients and methods}

\section{Study population and participants}

All subfertile women who started IVF in Amsterdam UMC, Vrije Universiteit Amsterdam, Netherlands, between January 2008 and March 2012 with a follow-up until 2014, were retrospectively reviewed. Data were obtained from paper, electronic patient files and, in case of ongoing pregnancy, via a routine patient questionnaire.

Inclusion criteria: women between 18 and 43 years old, trying to conceive for at least 1 year; TSH value measured preceding the first stimulation and embryo transfer.
Indications for IVF were (1) tubal occlusion, (2) severe endometriosis (grade (gr) III-IV) and (3) failed IUI for unexplained subfertility, mild male factor or mild endometriosis (gr I-II).

Exclusion criteria: (1) women treated with ICSI (semen $<2$ million spermatozoa per $\mathrm{mL}$ in diagnostic semen analysis; previous fertilization failure), (2) TSH out of reference value $(<0.3 \mathrm{mIU} / \mathrm{L}$ and $>4.5 \mathrm{mIU} / \mathrm{L})$, (3) a history of thyroid dysfunction or thyroid hormone substitution and (4) use of third-party gametes/surrogacy.

For patient characteristics, TSH values as well as data on BMI and tobacco and alcohol use nearest to and preceding the first cycle of IVF treatment were collected. A third-generation TSH assay (ECLIA Roche Cobas 8000) was used, with a reference range of $0.3-4.5 \mathrm{mIU} / \mathrm{L}$.

The following confounders were defined: age (years), primary or secondary subfertility (absence or presence of a previous clinical pregnancy), ethnicity (Caucasian/ other) (Benhadi et al. 2007, Korevaar et al. 2013, DhillonSmith et al. 2020), BMI (<20.9/21.0-28.9/29-34.9/>35 $\mathrm{kg} / \mathrm{m}^{2}$ (van der Steeg et al. 2008)), use of alcohol (no alcohol (0 units a week)/moderate (1-8 units a week)/heavy (>8 units a week) (Rachdaoui \& Sarkar 2013)) and tobacco (yes/ no (Hornstein 2016)).

Diminished ovarian reserve (FSH $>10 \mathrm{IU} / \mathrm{L}$ with an ovulating cycle) is associated with thyroid dysfunction (Chang et al. 2018). While looking for differences in indications, we did not adjust our analyses for this subfertility indication.

\section{In vitro fertilization data and pregnancy protocol}

Patients underwent the IVF protocol as outlined by Vergouw et al. (Vergouw et al. 2012). No assisted hatching was performed. Mean number of oocytes, fertilization rate (FR), number of fertilized oocytes (2PN), percentage of performed single embryo transfer (SET), embryo quality (EQ) in categories of good, medium and poor, following the Istanbul consensus scoring system (Alpha Scientists in Reproductive and Embryology 2011) were retrieved from the database, as well as mean number of cycles until first ongoing pregnancy or end of treatment, stimulation duration, sperm count in categories $(0.5-1.99,2-4.99$, 5-29.9 and > 30 million sperm cells) and percentage of endometrial thickness $>7 \mathrm{~mm}$ (last four parameters not shown). The local protocol in the analyzed years was to perform a SET in the first treatment including frozen embryo transfers when age $<38$ years and perform a double embryo transfer (DET) in others when embryo quality was medium or poor, or in the second and subsequent

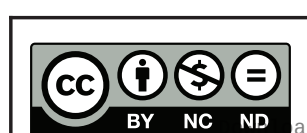

This work is licensed under a Creative Commons Attribution-NonCommercial-NoDerivatives 4.0 International License. ded from Bioscientifica.com at 04/26/2023 01:13:25PM 
Table 1 Patient characteristics of IVF-treated women. Data are presented as $n$ (\%), median (IQR) or as mean \pm S.D.

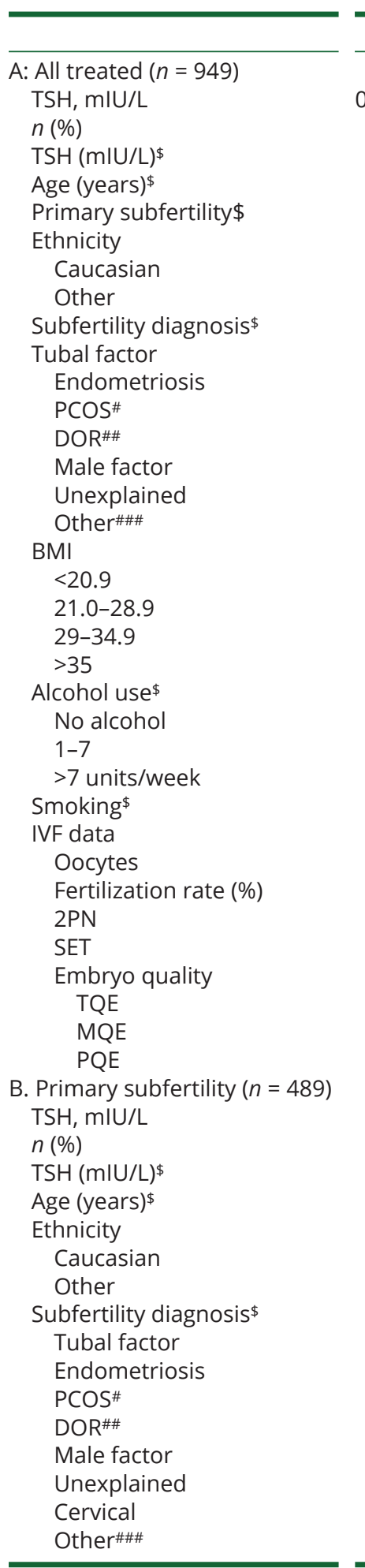

\begin{tabular}{|c|}
\hline I \\
\hline $0.30-1.21$ \\
\hline $239(25.2)$ \\
\hline $0.96(0.76-1.1)$ \\
\hline $35.5 \pm 4.4$ \\
\hline $119(49.8 \%)$ \\
\hline 180 (81.1\%) \\
\hline $42(18.9 \%)$ \\
\hline $60(25.1 \%)$ \\
\hline $40(16.7 \%)$ \\
\hline $7(2.9 \%)$ \\
\hline $9(3.8 \%)$ \\
\hline $20(8.4 \%)$ \\
\hline $72(30.1 \%)$ \\
\hline $10(4.2 \%)$ \\
\hline $74(31.1 \%)$ \\
\hline $136(57.1 \%)$ \\
\hline $23(9.7 \%)$ \\
\hline $5(2.1 \%)$ \\
\hline $90(37.7 \%)$ \\
\hline 125 (52.3\%) \\
\hline $24(10 \%)$ \\
\hline $57(23.8 \%)$ \\
\hline $9(6-15)$ \\
\hline $66(50-80)$ \\
\hline $6(3-10)$ \\
\hline 376 (68.6\%) \\
\hline 366 (66.3\%) \\
\hline $170(30.8 \%)$ \\
\hline $14(2.5 \%)$ \\
\hline $0.30-1.23$ \\
\hline $123(25.2 \%)$ \\
\hline $1.00(0.84-1.12)$ \\
\hline $34.2 \pm 4.6$ \\
\hline 98 (84.5\%) \\
\hline $18(15.5 \%)$ \\
\hline $23(18.7 \%)$ \\
\hline $27(22 \%)$ \\
\hline $4(3.3 \%)$ \\
\hline $4(3.3 \%)$ \\
\hline $11(8.9 \%)$ \\
\hline 39 (31.7\%) \\
\hline $7(5.7 \%)$ \\
\hline $4(3.3 \%)$ \\
\hline
\end{tabular}

\begin{tabular}{c}
\hline II \\
\hline $1.22-1.68$ \\
$236(24.9)$ \\
$1.46(1.32-1.57)$ \\
$35.9 \pm 4.3$ \\
$112(47.5 \%)$ \\
\\
$187(83.5 \%)$ \\
$37(16.5 \%)$ \\
\\
$48(20.3 \%)$ \\
$36(15.3 \%)$ \\
$9(3.8 \%)$ \\
$7(3.0 \%)$ \\
$28(11.9 \%)$ \\
$71(30.1 \%)$ \\
$11(4.7 \%)$ \\
$53(22.7 \%)$ \\
$154(66.1 \%)$ \\
$19(8.2 \%)$ \\
$7(3.0 \%)$
\end{tabular}

\begin{tabular}{c}
\hline III \\
\hline $1.69-2.31$ \\
$241(25.4)$ \\
$1.99(1.84-2.13)$
\end{tabular}

\begin{tabular}{cll} 
IV & & \multicolumn{1}{c}{$\boldsymbol{P}$} \\
\hline $2.32-4.50$ & & \\
$233(24.6)$ & & \\
$2.87(2.52-3.5)$ & & \\
$36 \pm 4.2$ & & $0.52^{* *}$ \\
$142(60.9 \%)$ & & 0.01 \\
& & 0.33
\end{tabular}

$178(81.3 \%)$

168 (76.7\%)

51 (23.3\%)

47 (19.5\%)

30 (12.4\%)

7 (2.9\%)

$10(4.1 \%)$

37 (15.4\%)

81 (33.6\%)

8 (3.3\%)

$70(29.4 \%)$

$143(60.1 \%)$

$19(8 \%)$

$6(2.5 \%)$

$97(41.3 \%)$

129 (54.9\%)

$9(3.8 \%)$

39 (16.5\%)

94 (39.3\%)

131 (54.8\%)

14 (5.9\%)

$34(14.2 \%)$

$8(5-14)$

$66(50-78)$

$5(3-10)$

309 (67.5\%)

308 (66.5\%)

$142(30.7 \%)$

$8(1.7 \%)$

$$
\begin{gathered}
9(6-16) \\
66(50-78) \\
6(3-10)
\end{gathered}
$$

408 (69.6\%)

396 (67.3\%)

$177(30.1 \%)$

$13(2.2 \%)$

0.51

45 (19.3\%)

$28(12.0 \%)$

7 (3.0\%)

7 (3.0\%)

$31(13.3 \%)$

91 (39.1\%)

$6(2.6 \%)$

$58(25.2 \%)$

$146(63.5 \%)$

$24(10.4 \%)$

2 (0.9\%)

87 (37.7\%)

$130(56.3 \%)$

$14(6.1 \%)$

25 (10.8\%)

0.36

0.39

0.93

0.87

0.12

0.13

0.64

0.41

$1.75-2.43$

1.24-1.74

$123(25.2 \%)$

$1.52(1.36-1.62)$

$34.6 \pm 4.5$

$10(6-16)$

$65.5(50-80)$

$6(3-10)$

$351(63.7 \%)$

$122(24.9 \%)$

$2.02(1.90-2.25)$

$35.1 \pm 3.6$

103 (89.6\%)

$12(10.4 \%)$

$93(83 \%)$

$19(17 \%)$

20 (16.3\%)

$27(22 \%)$

$4(3.3 \%)$

$4(3.3 \%)$

$16(13 \%)$

$34(27.6 \%)$

$5(4.1 \%)$

$5(4.1 \%)$

$11(9 \%)$

$20(16.4 \%)$

$6(4.9 \%)$

$4(3.3 \%)$

17 (13.9\%)

$50(41 \%)$

$4(3.3 \%)$

$5(4.1 \%)$

360 (64.9\%)

$172(31 \%)$

$23(4.1 \%)$

2.44-4.50

$121(24.7 \%)$

3.09 (2.67-3.66)

$35.6 \pm 4.3$

0.06 **

$90(76.9 \%)$

$27(23.1 \%)$

0.21

0.001

$0.41^{*}$

$0.95^{*}$

0.28

0.01

0.12

0.08

$\begin{array}{ll}21(17.4 \%) & 0.15 \\ 18(14.9 \%) & 0.35\end{array}$

$18(14.9 \%) \quad 0.35$

$4(3.3 \%) \quad 0.87$

$4(3.3 \%)$

$10(8.3 \%)$

1

$54(44.6 \%)$

0.39

$6(5 \%)$

0.02

0.82

$3(2.5 \%)$

0.89

(c) 2021 The authors Published by Bioscientifica Ltd

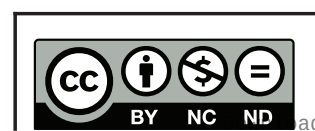

This work is licensed under a Creative Commons Attribution-NonCommercial-NoDerivatives 4.0 International License. 
Table 1 Continued.

\begin{tabular}{|c|c|c|}
\hline & $\mathbf{I}$ & II \\
\hline \multicolumn{3}{|l|}{ BMI } \\
\hline$<20.9$ & $47(38.2 \%)$ & $34(28.1 \%)$ \\
\hline $21.0-28.9$ & $65(52.8 \%)$ & $79(65.3 \%)$ \\
\hline $29-34.9$ & $11(8.9 \%)$ & $7(5.8 \%)$ \\
\hline$>35$ & 0 & $1(0.8 \%)$ \\
\hline \multicolumn{3}{|l|}{ Alcohol use } \\
\hline No alcohol & $41(33.3 \%)$ & $54(44.3 \%)$ \\
\hline $1-7$ & $72(58.5 \%)$ & $63(51.6 \%)$ \\
\hline$>7$ units/week & $10(8.1 \%)$ & $5(4.1 \%)$ \\
\hline Smoking\$ & $30(24.4 \%)$ & $22(17.9 \%)$ \\
\hline \multicolumn{3}{|l|}{ IVF data } \\
\hline Fertilization rate (\%) & $62(50-77)$ & $66(50-76)$ \\
\hline SET & $94(80.3 \%)$ & $94(82.5 \%)$ \\
\hline \multicolumn{3}{|l|}{ Embryo quality } \\
\hline TQE & $187(66.8 \%)$ & $157(66.8 \%)$ \\
\hline MQE & $89(31.8 \%)$ & $76(32.3 \%)$ \\
\hline PQE & $4(1.4 \%)$ & $1(0.4 \%)$ \\
\hline \multicolumn{3}{|c|}{ C: Secondary Subfertility $(n=460)$} \\
\hline $\mathrm{TSH}, \mathrm{mIU} / \mathrm{L}$ & $0.30-1.19$ & $1.20-1.635$ \\
\hline$n(\%)$ & $116(25.2 \%)$ & $114(24.8 \%)$ \\
\hline $\mathrm{TSH}(\mathrm{mIU} / \mathrm{L})^{\$}$ & $0.93(0.69-0.93)$ & $1.40(1.31-1.49)$ \\
\hline Age (years) $\$$ & $36.6 \pm 3.9$ & $37.4 \pm 3.6$ \\
\hline \multicolumn{3}{|l|}{ Ethnicity } \\
\hline Caucasian & $82(77.4 \%)$ & $85(77.3 \%)$ \\
\hline Other & $24(22.6 \%)$ & $25(22.7 \%)$ \\
\hline \multicolumn{3}{|l|}{ Subfertility diagnosis $\$$} \\
\hline Tubal factor & $38(32.8 \%)$ & $28(24.6 \%)$ \\
\hline Endometriosis & $12(10.3 \%)$ & $11(9.6 \%)$ \\
\hline PCOS\# & $3(2.6 \%)$ & $4(3.5 \%)$ \\
\hline DOR\#\# & $5(4.3 \%)$ & $3(2.6 \%)$ \\
\hline Male factor & $9(7.8 \%)$ & $11(9.6 \%)$ \\
\hline Unexplained & $33(28.4 \%)$ & $37(32.5 \%)$ \\
\hline Other\#\#\# & $5(4.3 \%)$ & $8(7.0 \%)$ \\
\hline \multicolumn{3}{|l|}{$\mathrm{BMI}$} \\
\hline$<20.9$ & $27(23.5 \%)$ & $20(17.7 \%)$ \\
\hline $21.0-28.9$ & $72(62.6 \%)$ & $73(64.6 \%)$ \\
\hline $29-34.9$ & $11(9.6 \%)$ & $14(12.4 \%)$ \\
\hline$>35$ & $5(4.3 \%)$ & $6(5.3 \%)$ \\
\hline \multicolumn{3}{|l|}{ Alcohol use $\$$} \\
\hline No alcohol & $48(41.4 \%)$ & $47(41.2 \%)$ \\
\hline $1-7$ & $55(47.4 \%)$ & $61(53.5 \%)$ \\
\hline$>7$ units/week & $13(11.2 \%)$ & $6(5.3 \%)$ \\
\hline Smoking\$ & $28(24.1 \%)$ & $17(14.9 \%)$ \\
\hline \multicolumn{3}{|l|}{ IVF data } \\
\hline Fertilization rate (\%) & $66(50-83.5)$ & $65(50-83)$ \\
\hline SET & $178(66.9 \%)$ & $137(62.5 \%)$ \\
\hline \multicolumn{3}{|l|}{ Embryo quality } \\
\hline TQE & $181(66.1 \%)$ & $143(63.8 \%)$ \\
\hline MQE & $82(29.9 \%)$ & $70(31.3 \%)$ \\
\hline $\mathrm{PQE}$ & $9(3.3 \%)$ & $7(3.1 \%)$ \\
\hline \multicolumn{3}{|c|}{ D: primary unexplained subfertile $(n=177)$} \\
\hline TSH, mIU/L & $0.30-1.31$ & $1.32-1.90$ \\
\hline$n(\%)$ & $44(24.9 \%)$ & $46(25.4 \%)$ \\
\hline $\mathrm{TSH}(\mathrm{mIU} / \mathrm{L})^{\$}$ & $1.02(0.86-1.18)$ & $1.67(1.49-1.82)$ \\
\hline Age (years) $\$$ & $35.8 \pm 4.1$ & $36 \pm 4.0$ \\
\hline Ethnicity & & \\
\hline
\end{tabular}

\begin{tabular}{c}
\hline III \\
\hline $33(27.7 \%)$ \\
$78(65.5 \%)$ \\
$7(5.9 \%)$ \\
$1(0.8 \%)$ \\
$36(30 \%)$ \\
$71(59.2 \%)$ \\
$13(10.8 \%)$ \\
$21(17.5 \%)$ \\
$66(50-77)$ \\
$94(82.5 \%)$ \\
$200(68.3 \%)$ \\
$86(29.4 \%)$ \\
$6(2.0 \%)$
\end{tabular}

1.64-2.22

$116(25.2 \%)$

$1.93(1.76-2.07)$

$36.9 \pm 3.7$

$77(78.6 \%)$

$21(21.4 \%)$

$33(28.4 \%)$

$9(7.8 \%)$

$3(2.6 \%)$

$5(4.3 \%)$

$16(13.8 \%)$

$34(29.3 \%)$

$3(2.6 \%)$

$31(27.2 \%)$

$69(60.5 \%)$

$11(9.6 \%)$

$3(2.6 \%$

$48(41.7 \%)$

$64(55.7 \%)$

$3(2.6 \%)$

$14(12.2 \%)$

66 (50-77)

$181(67 \%)$

$178(65.7 \%)$

$84(31 \%)$

$8(3 \%)$

1.91-2.59

$43(24.8 \%)$

$2.27(2.02-2.41)$

$36.1 \pm 3.3$

\begin{tabular}{c} 
IV \\
\hline $38(31.4 \%)$ \\
$72(59.5 \%)$ \\
$10(8.3 \%)$ \\
$1(0.8 \%)$
\end{tabular}

$46(38 \%)$

$68(56.2 \%)$

7 (5.8\%)

$12(9 . \% 9)$

0.03

$62(48.5-80) \quad 0.87$

$88(80.7 \%) \quad 0.43$

0.02

$155(58.5 \%)$

$97(36.6 \%)$

$13(4.9 \%)$

$2.23-4.50$

$114(24.8 \%)$

$2.64(2.34-3.13)$

$36.7 \pm 4.1$

$0.45^{\text {** }}$

0.97

$77(75.5 \%)$

$25(24.5 \%)$

$26(22.8 \%)$

$10(8.8 \%)$

$2(1.8 \%)$

$4(3.5 \%)$

$26(22.8 \%)$

$34(29.8 \%)$

$2(1.8 \%)$

$25(22.1 \%)$

$71(62.8 \%)$

$14(12.4 \%)$

$3(2.7 \%)$

$48(42.5 \%)$

$61(54 \%)$

$4(3.5 \%)$

$11(9.7 \%)$

$68(74.8-81) \quad 0.53$

$188(63.5 \%) \quad 0.68$

$213(72 \%)$

$73(24.7 \%)$

$10(3.4 \%)$

$2.60-4.50$

$44(24.9 \%)$

$3.10(2.82-3.64)$

$36.2 \pm 4.2$
0.015

0.55

0.33

0.89

0.88

0.86

0.004

0.88

0.20

0.90

0.15

0.68
0.27

$0.97^{* *}$

0.07 https://raf.bioscientifica.com

https://doi.org/10.1530/RAF-20-0065
(C) 2021 The authors Published by Bioscientifica Ltd

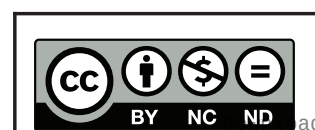

This work is licensed under a Creative Commons Attribution-NonCommercial-NoDerivatives 4.0 International License. 
Table 1 Continued.

\begin{tabular}{l}
\hline \\
\hline Caucasian \\
Other \\
Smoking $\$$ \\
IVF data \\
Fertilization rate (\%) \\
2PN \\
Embryo quality \\
TQE \\
MQE \\
PQE
\end{tabular}

\begin{tabular}{c}
\hline $\mathbf{I}$ \\
\hline $36(83.7 \%)$ \\
$7(16.3 \%)$ \\
$9(20.5 \%)$ \\
$66(50-88.8)$ \\
$5(1-9)$ \\
$22(36.7 \%)$ \\
$37(61.7 \%)$ \\
$37(1.7 \%)$
\end{tabular}

\begin{tabular}{c}
\hline II \\
\hline $40(95.2 \%)$ \\
$2(4.8 \%)$ \\
$7(15.2 \%)$ \\
$62(48.5-77.3)$ \\
$6(3-9)$
\end{tabular}

\begin{tabular}{c}
\hline III \\
\hline $37(90.2 \%$ \\
$4(9.8 \%)$ \\
$6(14.3 \%)$
\end{tabular}

\begin{tabular}{c}
\hline IV \\
\hline $33(76.7 \%)$ \\
$10(23.3 \%)$ \\
$4(9.1 \%)$
\end{tabular}

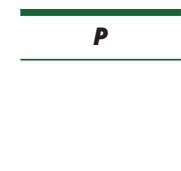

69(56.3-80.8) 6 (4-9)

64.5 (42.5-75.0) $5(2-10)$

34 (55.7\%)

$27(44.3 \%)$

0

$23(41.1 \%)$

$31(55.4 \%)$

$2(3.6 \%)$

$28(41.8 \%)$

$37(55.2 \%)$

2 (3.0\%)

*Kruskal-Wallis, **ANOVA; \#PCOS, polycystic ovary syndrome; \#\#DOR, diminished ovarian reserve, defined as ovulatory cycles with FSH > 10 U/L; \#\#Other: congenital uterus anomaly, Asherman's syndrome; SET, single embryo transfer; Embryo quality: top quality embryo, medium quality embryo; poor quality embryo (for statistical comparison and in order to objectively quantify the embryo quality, embryos were subdivided into three groups: top quality embryos, medium quality embryos and poor quality embryos. The embryos are mostly classified according to all criteria of the Istanbul consensus 2011 (Alpha Scientists in Reproductive and Embryology 2011). Top quality fresh embryos: $<10 \%$ fragmentation and 7, 8, 9 or 10 cells, compaction or morula. Top quality frozen-thawed embryos: blastocyst, expended, hatching or hatched blastocyst. Medium quality fresh embryos: 10-50\% fragmentation and 7, 8, 9 or 10 cells, compaction or morula; or $<10 \%$ fragmentation and 5 or 6 cells. Medium quality frozen-thawed embryos: compaction, early blastocyst. Poor quality fresh embryos: $>10 \%$ fragmentation and 5 or 6 cells, or $>50 \%$ fragmentation in $<5$ cells. Poor quality frozen-thawed embryos: $7,8,9,10$ cells, morula or early compaction). ${ }^{\$}$ First cycle per person, in case of $<2 \times 10^{6}$, a second sample is asked for.

treatment. Involved couples with sperm count below 2 million sperm cells did not agree on ICSI treatment.

Clinical pregnancy was defined as an intrauterine gestation at 6-8 weeks gestation. Ongoing pregnancy was defined as an intrauterine gestation with beating heart, at 11 weeks amenorrhea. Pregnancy loss was defined as a non-viable clinical pregnancy. Live birth was defined as a healthy child born $>24$ weeks.

\section{Statistical analysis}

SPSS 26 was used for statistical analysis. Demographic and baseline characteristics were compared between groups using one-way ANOVA, Kruskal-Wallis ANOVA and chisquare test. Live birth rate, clinical and ongoing pregnancy were determined with patient as the unit of analysis, that is, data are shown for cumulative cycles until ongoing pregnancy in percentages per patient, and with first cycle as the unit of analysis. Pregnancy loss is the percentage per clinical pregnancy. Patients were equally categorized into four groups each covering $25 \%$ based on TSH values for uncovering possible $\mathrm{u}$-shaped associations of TSH and pregnancy outcomes, such as has been described between thyroid hormone levels and IQ levels of the child (Korevaar et al. 2016). Subgroup analyses were performed if significant differences in the distribution of the characteristics across TSH quartiles were found. Of each subgroup, specific interquartile cut-off values were determined. In the analyses, the upper TSH quartile (group IV) was used as a reference. Differences in pregnancy outcomes based on interquartile TSH groups were tested using logistic regression analysis. Adjusted analyses were performed in which we corrected for age, primary or secondary subfertility, ethnicity, BMI, use of alcohol and tobacco. Odds ratios were reported as effect-size together with their 95\% CI and $P$-values.

\section{Results}

\section{Patient characteristics}

Nine hundred forty-nine women met the inclusion criteria. The interquartile TSH groups of the overall IVF-treated women (Table 1A) were as follows, I: 0.3-1.21 mIU/L; II: 1.22-1.68 mIU/L; III: 1.69-2.31 mIU/L and IV: 2.32-4.5 $\mathrm{mIU} / \mathrm{L}$. In the distribution of TSH (Fig. 1), the bottom three quartiles correspond to low-normal TSH levels, 0.3-2.5 $\mathrm{mIU} / \mathrm{L}$, while the upper quartile corresponded to the 'tail' of the right-skewed TSH distribution, with its lower cut-off level close to the discussed upper TSH reference level of $2.5 \mathrm{mIU} / \mathrm{L}$ for women with subfertility.

Age, subfertility diagnosis, ethnicity, BMI and alcohol use were comparable in the four TSH quartiles (Table 1). The percentage of primary subfertile women $(n=489)$ was higher in upper quartile TSH: $60.9 \%$ compared to about 50\% in the lower three TSH quartiles (I: 119 (49.8\%); II: 112 (47.5\%); III: 116 (48.1\%); IV: 142 (60.9\%); P=0.01). A trend of a larger percentage of women with unexplained subfertility ( $n=315)$ was found in upper quartile TSH: I: 72 (30.1\%); II: 71 (30.1\%); III: 81 (33.6\%); IV: 91 (39.1\%); 


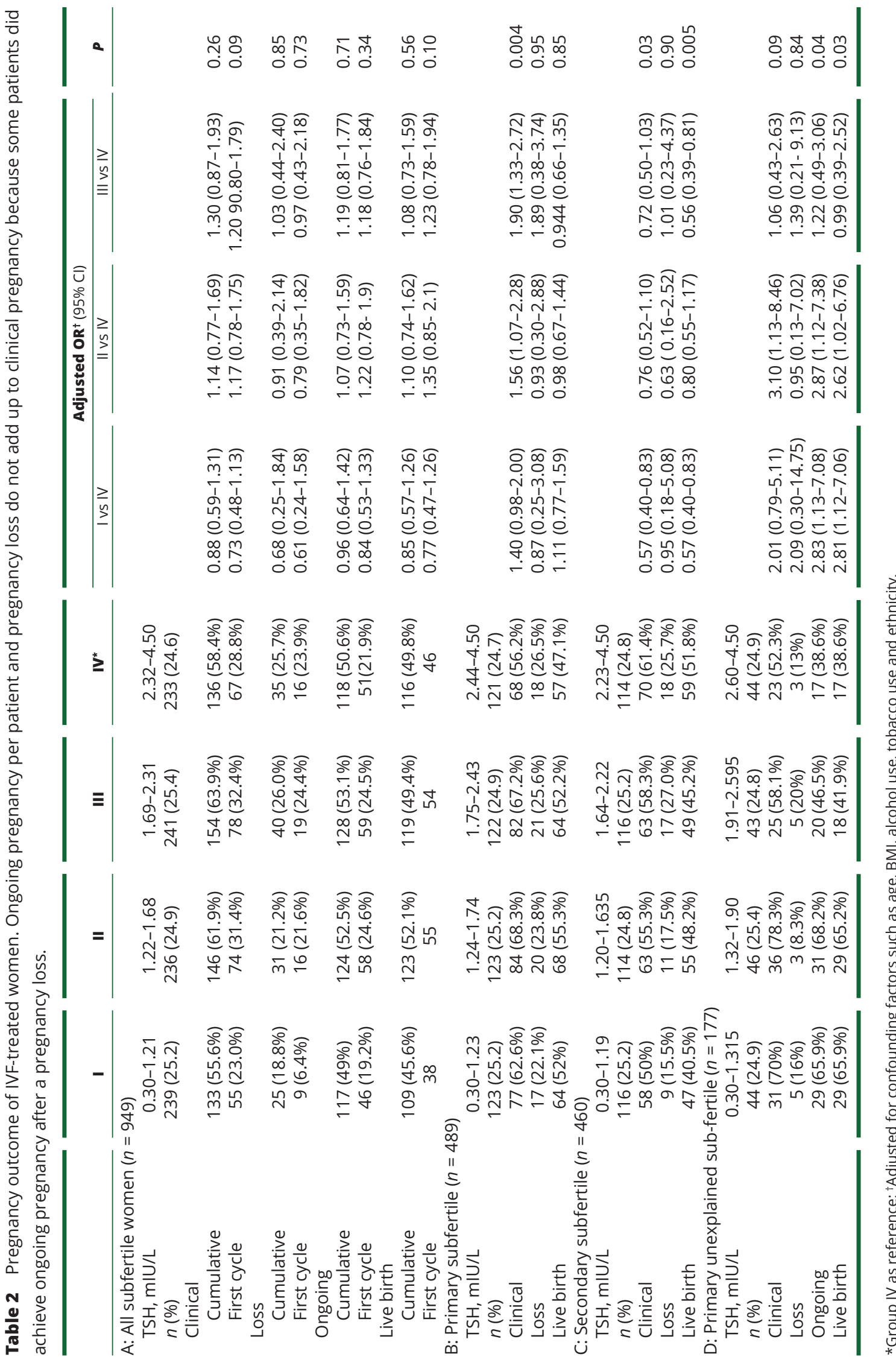




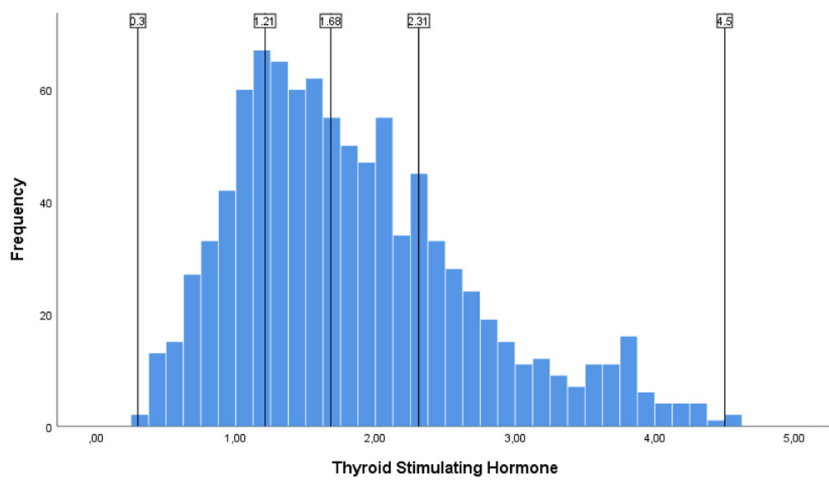

Figure 1 Distribution of TSH of 949 IVF treated women.

$P=0.13)$. Smokers were more prevalent in the lowest TSH quartile group (I: 57 (23.8\%); II: 39 (16.5\%); III: 34 (14.2\%); IV: $25(10.8 \%) ; P=0.001)$.

Subgroup analysis of primary $(n=489$, Table 1B) and secondary subfertile women $(n=460$, Table 1C) was performed. Age tended to be higher in primary subfertile women within upper quartile TSH (I: 34.2; II: 34.6; III 35.1; IV: $35.6 ; P=0.06)$. The percentage of primary subfertile women with unexplained subfertility $(n=177$, Table $1 \mathrm{D})$ was higher in the upper TSH quartiles (I: 39 (31.7\%); II: 34 (27.6\%); III: 50 (41\%); IV: 54 (44.6\%); $P=0.02)$, not associated with age or BMI. The percentage of secondary subfertile women with male factor subfertility $(n=62)$ increased toward upper TSH quartiles (I: 9 (7.8\%); II: 11 (9.6\%); III: 16 (13.8\%); IV: 26 (22.8\%); $P=0.004)$. In both primary $(P=0.03)$ and secondary subfertile women $(P=0.02)$, smokers were more represented in the lowest TSH quartile group.

\section{In vitro fertilization data}

No U-shaped results were revealed for IVF data. In Table 1A, showing all women, the number of oocytes, fertilization rate, number of fertilized oocytes and embryo quality were not different over the four TSH quartiles. Single embryo transfers (SET) were less often performed in upper TSH quartile: $63.7 \%$ compared to an average of $67 \%$ in the lower quartiles $(P=0.01)$.

In primary subfertile women (Table 1B), the percentage of top quality embryo's (TQE) was lower in the upper TSH quartile (I: $66.8 \%$; II: $66.8 \%$; III: 68.3\%; IV: $58.5 \%$; $P=0.02$ ). This difference in distribution was not found in primary unexplained subfertile women (Table 1C), although the overall percentage of TQE in primary unexplained subfertile women was lower, being $37-56 \%$, compared to
$59-68 \%$ in primary subfertile women, and to $64-72 \%$ in secondary subfertile women.

No other differences in distribution were found (Table $1 \mathrm{~B}$ nor $\mathrm{C}$ ), especially not in the number of oocytes or in fertilization rate.

\section{Pregnancy outcomes}

No U-shaped results were revealed for pregnancy outcomes. All results were adjusted for confounders. Cumulative pregnancy outcomes across TSH quartiles in all IVF-treated women (Table 2A) were not different. In primary subfertile IVF-treated women, clinical pregnancy was significantly lower in women with high-normal TSH (Table 2B). In primary unexplained subfertile IVF-treated women (Table 2D), live birth rate, clinical and ongoing pregnancy rate were lower in the two top TSH quartiles $(n=87 / 177)$ compared to the two bottom quartiles ( $n=177$ live birth rate: I: 29 (65.9\%); II: 29 (65.2\%); III: 18 (41.9\%); IV: 17 (38.6\%); $P=0.03)$. Odds ratios for live birth rate were more than two-fold, adjusted for confounders (age, BMI, alcohol use, tobacco use and ethnicity) for women with TSH $<1.9 \mathrm{mIU} / \mathrm{L}$ compared to women with TSH 2.6-4.5 mIU/L (group I-IV 2.18 (C.I. 1.127.06) and group II-IV: 2.62 (C.I. 1.02-6.76); $P=0.01$ ).

With secondary subfertility, a significantly lower clinical and live birth rate in the lowest TSH quartile were found (Table 2C).

Because of the limited number of women treated for male factor subfertility $(n=62)$, no reliable subanalyses on fertility outcome could be performed.

\section{Discussion}

Based on the overall results of this large single-center IVF cohort study, we conclude that high-normal compared to low-normal levels of TSH are not associated with adverse implications for pregnancy outcomes in the overall group of conventional IVF-treated women. It is therefore possible that minor changes in thyroid status may not be associated with fertility outcomes. However, higher TSH levels are associated with primary subfertility. In subgroupanalysis of primary subfertile women, more unexplained subfertility was revealed in the upper TSH quartiles. These primary unexplained subfertile women, $9.2 \%$ of this cohort ( $n=87 / 949$ ), had significantly lower odds for cumulative live birth rate, as well as for clinical and ongoing pregnancy after IVF.

In secondary subfertile women in the upper normal TSH quartile, male factor subfertility dominated, with

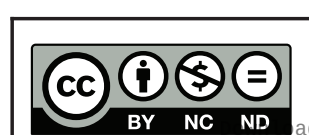

This work is licensed under a Creative Commons Attribution-NonCommercial-NoDerivatives 4.0 International License. 
higher live birth rates. Of interest were the lower live birth rates in the lowest TSH group. The large amount of smokers was remarkable, and, though adjusted for smoking, the fewer births could still have been partly due to smoking habits.

Primary and secondary subfertile women have not often been analyzed separately. These unexpected findings bring us to the question: 'Why did primary subfertile women more often show high-normal TSH levels?' We can only speculate for a plausible explanation. Aside from coincidence, we believe the following may have happened: the included women had no clinical or biochemical signs of any thyroid dysfunction according to the inclusion criteria. Since thyroid dysfunction often reveals itself during or shortly after pregnancy, the possibility exists that our subgroup of secondary subfertile women, in contrast to the group of primary subfertile women, was devoid of women with borderline clinical hypothyroidism. The difference in TSH distribution between women with primary and secondary subfertility could therefore be explained by selection bias, as in primary subfertility, thyroid dysfunction may have gone undetected until subfertility presented itself as a symptom. In secondary subfertile women, on the contrary, subclinical thyroid dysfunction had already manifested itself during or after the challenge of a previous pregnancy.

TSH reference ranges vary with ethnicity: Caucasian women have slightly higher TSH reference levels than African and Asian women (Benhadi et al. 2007, Korevaar et al. 2013, Dhillon-Smith et al. 2020). Nevertheless, in our cohort with the majority of our patients being Caucasian, a higher percentage in the upper quartile TSH was nonCaucasian. What impact this has on fewer live births remains to be determined.

In accordance with previously described age-dependent TSH increase (Baloch et al. 2003), the distribution of age showed a trend toward a higher age in the upper quartile TSH group in primary subfertile women, though not in primary unexplained subfertile women nor in secondary subfertile women. In the latter group, however, age was higher in all TSH groups. In accordance with previously described smoking-dependent TSH decrease via the adrenergic pathway (Wiersinga 2013), the percentage of women smoking cigarettes was distributed inversely to the TSH levels in all (sub)analyses. For these reasons, our study adjusted for these factors. The significantly reduced embryo quality in primary subfertile women in the upper quartile TSH group was in agreement with the lower percentage of performed single embryo transfers (SET), according to the protocol at that time. Analyzing the number of SET in the first cycle only, this difference indeed was not seen.

Live birth rate in primary subfertile women was $5 \%$ lower in upper quartile TSH compared to the lower three TSH quartiles, though not significant. Live birth rate in primary unexplained subfertile women however was distributed significantly different, with a $15 \%$ lower live birth rate in the top two TSH quartiles.

An association of high-normal TSH levels in women with primary unexplained subfertility and fertility outcome might disclose subtle thyroid dysfunction contributing to subfertility. Subclinical hypothyroidism can affect early embryo development (Karmon et al. 2016, Poppe et al. $2018 a$ ). In our cohort, a reduced embryo quality was seen in the upper quartile TSH group of primary subfertile women. As mentioned, we know from the recently published tablet Trial that $9.5 \%$ of subfertile women have thyroid peroxidase antibodies (TPOAbs), as do $20.1 \%$ of subfertile women with TSH $>2.5 \mathrm{mIU} / \mathrm{L}$ (Dhillon-Smith et al. 2020). Monteleone et al. (2011) and Poppe et al. (2018a) describe hampering of fertilization or early embryo development associated with thyroid autoimmunity, although others do not support this (Alexander et al. 2017). TPOAb-positive women who undergo IVF, amount a high TSH response during ovarian stimulation, possibly contributing to poorer egg quality and thus lowering live birth rate.

Nevertheless, the hypothesized pathophysiologic role of the thyroid in subfertility is shifting from thyroid hypofunction reflected in an increased TSH level to thyroid autoimmunity (TAI) (Weghofer et al. 2015). Via an enhanced global autoimmune state (Thangaratinam et al. 2011), TAI can have an adverse effect on placental and fetal development (Thangaratinam et al. 2011). Furthermore, TPO antibodies are assumed to (cross-)react with the zona pellucida, hampering fertilization and/ or early embryo development (Andrisani et al. 2018, Poppe et al. 2018a). Autoimmunity comes to expression with increasing age, often from the third decade. It can develop slowly to autoimmune-related subtle thyroid dysfunction, perhaps at first only affecting fertility (Simopoulou et al. 2019) or fertilization. In the decision to perform conventional IVF or ICSI in women of advanced maternal age (Tannus et al. 2017, Poppe et al. 2021), thyroid dysfunction might be important.

Recently two randomized placebo-controlled trials were published in which TPOAb-positive women were randomized for the use of levothyroxine. Primary endpoint in the postal trial was miscarriage rate in subfertile women (Wang et al. 2017), and in the tablet trial, it was live birth rate in women with recurrent miscarriage and in subfertile

This work is licensed under a Creative Commons Attribution-NonCommercial-NoDerivatives 4.0 International License. International License.
ded from Bioscientifica.com at 04/26/2023 01:13:25PM 
women (Dhillon-Smith et al. 2019). Both studies showed no benefit of the randomized fixed dose of levothyroxine. In the tablet trial, the levothyroxine dose is suggested to be too low, as in both groups around 10\% developed abnormal thyroid function tests during pregnancy. Importantly, in relation to our study: in both trials, IVF and ICSI are distributed equally over the levothyroxine and placebo group, but the separate IVF vs ICSI outcomes were not analyzed. As suggested by Poppe in the recent ETA guidelines (Poppe et al. 2021), the treatment for subfertile TPOAb positive women might be ICSI instead of IVF, overcoming hampered fertilization and early embryo development.

\section{Strengths and weaknesses}

Thyroid peroxidase antibodies were not measured in our clinic, following NICE and International Guidelines (Practice Committee of the American Society for Reproductive 2015, Alexander et al. 2017), and neither was FT4, though suggested in the ETA guidelines 2021 (Poppe et al. 2021). This weakens the clinical interpretability where the role of thyroid autoimmunity in subtle hypothyroidism and subfertility can only be hypothesized.

A strength of this study however is the size of the cohort including only conventional IVF-treatments, with sufficient numbers to analyze primary and secondary subfertile women separately. The distinction of primary especially unexplained subfertile women as a subgroup supports further exploring thyroid autoimmunity and subtle thyroid dysfunction as a pathophysiological mechanism hampering fertilization and early embryo development.

\section{Conclusion}

In this study, TSH levels between 2.5 and $4.5 \mathrm{mIU} / \mathrm{L}$ were not associated with different fertility outcomes for the majority of women treated with coventional IVF. An exception can be made for $9 \%$ of these women $(n=87 / 949)$ with primary unexplained subfertility, who had $15 \%$ lower live birth rate associated with high-normal TSH.

\section{Declaration of interest}

The authors declare that there is no conflict of interest that could be perceived as prejudicing the impartiality of the research reported.

\section{Funding}

This work did not receive any specific grant from any funding agency in the public, commercial or not-for-profit sector.

\section{Ethical approval}

This study has been formally exempted from ethical approval granted by the Institutional Review Board of the VU University Medical Centre (reference 2013.83, dated 25th March 2013).

\section{Availability of the data}

The datasets during and/or analysed during the current study available from the corresponding author on reasonable request.

\section{Author contribution statement}

$C R \vee D-D$ and $E W C M \vee D$ designed the study. $C R \vee D-D$ sought for ethical approval. C R v D-D, K A, M t H, Y F, A d R and G B collected the data. R S supervised the collection of the data. E W C M v D supervised the research and writing process. $P M \vee d V$ and $C R \vee D-D$ performed the statistical analysis. C B L was the supervisor of the study and of critical analysis, interpretation and presentation of the study. All authors gave full input to the final draft of the paper.

\section{References}

Alexander EK, Pearce EN, Brent GA, Brown RS, Chen H, Dosiou C, Grobman WA, Laurberg P, Lazarus JH, Mandel SJ, et al. 20172017 Guidelines of the American Thyroid Association for the diagnosis and management of thyroid disease during pregnancy and the postpartum. Thyroid 27 315-389. (https://doi.org/10.1089/ thy.2016.0457)

Alpha Scientists in Reproductive Medicine and ESHRE Special Interest Group of mbryology 2011. The Istanbul consensus workshop on embryo assessment: proceedings of an expert meeting. Human Reproduction 26 1270-1283.

Andrisani A, Sabbadin C, Marin L, Ragazzi E, Dessole F, Armanini D, Dona G, Bordin L \& Ambrosini G 2018 The influence of thyroid autoimmunity on embryo quality in women undergoing assisted reproductive technology. Gynecological Endocrinology 34 752-755. (https://doi.org/10.1080/09513590.2018.1442427)

Baloch Z, Carayon P, Conte-Devolx B, Demers LM, FeldtRasmussen U, Henry JF, Livosli VA, Niccoli-Sire P, John R, Ruf J, et al. 2003 Laboratory medicine practice guidelines. Laboratory support for the diagnosis and monitoring of thyroid disease. Thyroid 13 3-126. (https://doi. org/10.1089/105072503321086962)

Benhadi N, Wiersinga WM, Reitsma JB, Vrijkotte TG, van der Wal MF \& Bonsel GJ 2007 Ethnic differences in TSH but not in free T4 concentrations or TPO antibodies during pregnancy. Clinical Endocrinology 66 765-770. (https://doi.org/10.1111/j.13652265.2007.02803.x)

Chai J, Yeung WY, Lee CY, Li HW, Ho PC \& Ng HY 2014 Live birth rates following in vitro fertilization in women with thyroid autoimmunity and/or subclinical hypothyroidism. Clinical Endocrinology 80 122-127. 
Chang Y, Li J, Li X, Liu H \& Liang X 2018 Egg quality and pregnancy outcome in young infertile women with diminished ovarian reserve. Medical Science Monitor 24 7279-7284. (https://doi.org/10.12659/ MSM.910410)

Cramer DW, Sluss PM, Powers RD, Mcshane P, Ginsburgs ES, Hornstein MD, Vitonis AF \& Barbieri RL 2003 Serum prolactin and TSH in an in vitro fertilization population: is there a link between fertilization and thyroid function? Journal of Assisted Reproduction and Genetics 20 210-215. (https://doi.org/10.1023/a:1024151210536)

Dhillon-Smith RK, Middleton LJ, Sunner KK, Cheed V, Baker K, Farrell-Carver S, Bender-Atik R, Agrawal R, Bhatia K, Edi-Osagie E, et al. 2019 Levothyroxine in women with thyroid peroxidase antibodies before conception. New England Journal of Medicine 380 1316-1325. (https://doi.org/10.1056/NEJMoa1812537)

Dhillon-Smith RK, Tobias A, Smith PP, Middleton LJ, Sunner KK, Baker K, Farrell-Carver S, Bender-Atik R, Agrawal R, Bhatia K, et al. 2020 The prevalence of thyroid dysfunction and autoimmunity in women with history of miscarriage or subfertility. Journal of Clinical Endocrinology and Metabolism 105 dgaa302. (https:// doi.org/10.1210/clinem/dgaa302)

Hornstein MD 2016 Lifestyle and IVF outcomes. Reproductive Sciences 23 1626-1629. (https://doi.org/10.1177/1933719116667226)

Jatzko B, Vytiska-Bistorfer E, Pawlik A, Promberger R, Mayerhofer K \& Ott J 2014 The impact of thyroid function on intrauterine insemination outcome - a retrospective analysis. Reproductive Biology and Endocrinology 12 28. (https://doi. org/10.1186/1477-7827-12-28)

Karmon AE, Batsis M, Chavarro JE \& Souter I 2015 Preconceptional thyroid-stimulating hormone levels and outcomes of intrauterine insemination among euthyroid infertile women. Fertility and Sterility 103 258.e1-263.e1. (https://doi.org/10.1016/j.fertnstert.2014.09.035)

Karmon AE, Cardozo ER, Souter I, Gold J, Petrozza JC \& Styer AK 2016 Donor TSH level is associated with clinical pregnancy among oocyte donation cycles. Journal of Assisted Reproduction and Genetics 33 489-494. (https://doi.org/10.1007/s10815-016-0668-6)

Korevaar TI, Medici M, De Rijke YB, Visser W, De Muinck KeizerSchrama SM, Jaddoe VW, Hofman A, Ross HA, Visser WE, Hooijkaas H, et al. 2013 Ethnic differences in maternal thyroid parameters during pregnancy: the Generation R Study. Journal of Clinical Endocrinology and Metabolism 98 3678-3686. (https://doi. org/10.1210/jc.2013-2005)

Korevaar TI, Muetzel R, Medici M, Chaker L, Jaddoe VW, De Rijke YB, Steegers EA, Visser TJ, White T, Tiemeier H, et al. 2016 Association of maternal thyroid function during early pregnancy with offspring IQ and brain morphology in childhood: a population-based prospective cohort study. Lancet: Diabetes and Endocrinology 4 35-43. (https://doi.org/10.1016/S2213-8587(15)003277)

Michalakis KG, Mesen TB, Brayboy LM, Yu B, Richter KS, Levy M, Widra E \& Segars JH 2011 Subclinical elevations of thyroidstimulating hormone and assisted reproductive technology outcomes. Fertility and Sterility 95 2634-2637. (https://doi.org/10.1016/j. fertnstert.2011.02.056)

Monteleone P, Parrini D, Faviana P, Carletti E, Casarosa E, Uccelli A, Cela V, Genazzani AR \& Artini PG 2011 Female infertility related to thyroid autoimmunity: the ovarian follicle hypothesis. American Journal of Reproductive Immunology 66 108-114. (https://doi.org/10.1111/j.1600-0897.2010.00961.x)

Pekcan MK, Ozgu-Erdinc AS \& Yilmaz N 2019 Impact of subclinical hypothyroidism and thyroid autoimmunity on clinical pregnancy rate after intrauterine insemination in euthyroid women. JBRA Assisted Reproduction 23 137-142. (https://doi.org/10.5935/15180557.20190027)

Poppe K, Autin C, Veltri F, Kleynen P, Grabczan L, Rozenberg S \& Ameye L 2018 a Thyroid autoimmunity and intracytoplasmic sperm injection outcome: a systematic review and meta-analysis. Journal of
Clinical Endocrinology and Metabolism 1031755 - 1766. (https://doi. org/10.1210/jc.2017-02633)

Poppe K, Veltri F \& Autin C $2018 b$ Does levothyroxine improve pregnancy outcomes in euthyroid women with thyroid autoimmunity undergoing assisted reproductive technology? Thyroid Research 117. (https://doi.org/10.1186/s13044-018-0052-y)

Poppe K, Bisschop P, Fugazzola L, Minziori G, Unuane D \& Weghofer A 20212021 European Thyroid Association Guideline on thyroid disorders prior to and during assisted reproduction. European Thyroid Journal 9 281-295. (https://doi.org/10.1159/000512790)

Practice Committee of the American Society for Reproductive Medicine 2015 Subclinical hypothyroidism in the infertile female population: a guideline. Fertility and Sterility 104 545-553. (https://doi.org/10.1016/j. fertnstert.2015.05.028)

Rachdaoui N \& Sarkar DK 2013 Effects of alcohol on the endocrine system. Endocrinology and Metabolism Clinics of North America 42 593-615. (https://doi.org/10.1016/j.ecl.2013.05.008)

Reh A, Grifo J \& Danoff A 2010 What is a normal thyroid-stimulating hormone (TSH) level? Effects of stricter TSH thresholds on pregnancy outcomes after in vitro fertilization. Fertility and Sterility 94 2920-2922. (https://doi.org/10.1016/j.fertnstert.2010.06.041)

Repelaer van Driel-Delprat CC, Van Dam EWCM, Van De Ven PM, Homsma S, van der Kooij L, Vis E, Peeters RP, Schats R \& Lambalk CB 2019 Live birth rate after intrauterine insemination is not different between women with lower quartile versus higher quartile normal range thyroid stimulating hormone levels. Human Reproduction Open 2019 hoz002. (https://doi.org/10.1093/hropen/ hoz002)

Simopoulou M, Sfakianoudis K, Maziotis E, Grigoriadis S, Giannelou P, Rapani A, Tsioulou P, Pantou A, Kalampokas T, Vlahos N, et al. 2019 The impact of autoantibodies on IVF treatment and outcome: a systematic review. International Journal of Molecular Sciences 20 892. (https://doi.org/10.3390/ijms20040892)

Tan S, Dieterle S, Pechlavanis S, Janssen OE \& Fuhrer D 2014 Thyroid autoantibodies per se do not impair intracytoplasmic sperm injection outcome in euthyroid healthy women. European Journal of Endocrinology 170 495-500. (https://doi.org/10.1530/EJE-13-0790)

Tannus S, Son WY, Gilman A, Younes G, Shavit T \& Dahan MH 2017 The role of intracytoplasmic sperm injection in non-male factor infertility in advanced maternal age. Human Reproduction 32 119-124. (https://doi.org/10.1093/humrep/dew298)

Thangaratinam S, Tan A, Knox E, Kilby MD, Franklyn J \& Coomarasamy A 2011 Association between thyroid autoantibodies and miscarriage and preterm birth: meta-analysis of evidence. BMJ 342 d2616. (https://doi.org/10.1136/bmj.d2616)

Tuncay G, Karaer A, Inci Coskun E, Baloglu D \& Tecellioglu AN 2018 The impact of thyroid-stimulating hormone levels in euthyroid women on intrauterine insemination outcome. BMC Women's Health 18 51. (https://doi.org/10.1186/s12905-018-0541-0)

Unuane D, Velkeniers B, Bravenboer B, Drakopoulos P, Tournaye H, Parra J \& De Brucker M 2017 Impact of thyroid autoimmunity in euthyroid women on live birth rate after IUI. Human Reproduction 32 915-922. (https://doi.org/10.1093/humrep/dex033)

van der Steeg JW, Steures P, Eijkemans MJ, Habbema JD, Hompes PG, Burggraaff JM, Oosterhuis GJ, Bossuyt PM, van der Veen F \& Mol BW 2008 Obesity affects spontaneous pregnancy chances in subfertile, ovulatory women. Human Reproduction $\mathbf{2 3}$ 324-328. (https://doi.org/10.1093/humrep/dem371)

Vergouw CG, Kieslinger DC, Kostelijk EH, Botros LL, Schats R, Hompes PG, Sakkas D \& Lambalk CB 2012 Day 3 embryo selection by metabolomic profiling of culture medium with nearinfrared spectroscopy as an adjunct to morphology: a randomized controlled trial. Human Reproduction 27 2304-2311. (https://doi. org/10.1093/humrep/des175)

Wang H, Gao H, Chi H, Zeng L, Xiao W, Wang Y, Li R, Liu P, Wang C, Tian Q, et al. 2017 Effect of levothyroxine on miscarriage 
among women with normal thyroid function and thyroid autoimmunity undergoing in vitro fertilization and embryo transfer: a randomized clinical trial. JAMA 318 2190-2198. (https://doi. org/10.1001/jama.2017.18249)

Weghofer A, Himaya E, Kushnir VA, Barad DH \& Gleicher N 2015 The impact of thyroid function and thyroid autoimmunity on embryo quality in women with low functional ovarian reserve: a case-control study. Reproductive Biology and Endocrinology 13 43. (https://doi. org/10.1186/s12958-015-0041-0)

Wiersinga WM 2013 Smoking and thyroid. Clinical Endocrinology 79 145-151. (https://doi.org/10.1111/cen.12222)

Zhang C, Guo L, Zhu B, Feng Y, Yu S, An N \& Wang X 2013 Effects of 3,5,3'-triiodothyronine (t3) and follicle stimulating hormone on apoptosis and proliferation of rat ovarian granulosa cells. Chinese Journal of Physiology 56 298-305. (https://doi.org/10.4077/CJP.2013.BAB186)
Zhao T, Chen BM, Zhao XM \& Shan ZY 2018 Meta-analysis of ART outcomes in women with different preconception TSH levels. Reproductive Biology and Endocrinology 16 111. (https://doi.org/10.1186/ s12958-018-0424-0)

Zhong YP, Ying Y, Wu HT, Zhou CQ, Xu YW, Wang Q, Li J, Shen XT \& Li J 2012 Relationship between antithyroid antibody and pregnancy outcome following in vitro fertilization and embryo transfer. International Journal of Medical Sciences 9 121-125. (https:// doi.org/10.7150/ijms.3467)

Received in final form 13 October 2021

Accepted 15 October 2021

Accepted Manuscript published online 18 October 2021 (c) 2021 The authors Published by Bioscientifica Ltd
This work is licensed under a Creative Commons Attribution-NonCommercial-NoDerivatives 4.0 International License. Int from Bioscientifica, com at 04/26/2023 01:13:25PM 